\title{
Aberration generators in tandem
}

\author{
J. Ojeda-Castaneda, ${ }^{* 1}$ Ana L. Barragán-Chávez, ${ }^{1}$ and Cristina M. Gómez-Sarabia ${ }^{2}$ \\ ${ }^{1}$ Electronics Department, Campus Salamanca, University of Guanajuato, 36885 Guanajuato, México \\ ${ }^{2}$ Digital Arts, Campus Salamanca, University of Guanajuato, 36885 Guanajuato, México
}

Received February 20, 2015; accepted March 31, 2015; published March 31, 2015

\begin{abstract}
We discuss the use of a pair of phase masks, which have both radial and helical variations, for optically implementing a linear combination of two different types of wavefront aberrations. We show that by using two pairs of phase masks, one can combine symmetric and asymmetric aberrations. Some simple numerical simulations illustrate our proposed procedure.
\end{abstract}

For several optical applications it is convenient to have optical devices that can generate wave aberrations, with controllable aberration coefficients [1-2]. For this purpose, some authors have applied two phase conjugated masks; as proposed by Kitajima [3], Lohmann [4-7], Alvarez [8, 9] and Palusinski et al. [10].

Other methods incorporate two phase masks, which have phase variations expressible in terms of trigonometric functions of the polar angle $[11,12]$.

Rather recently, some of us have suggested the use of vortex phase pairs, which have linear phase variations in the polar angle $[13,14]$. In Table 1 , we summarize these efforts.

Table 1. Alternatives of phase angular variations.

\begin{tabular}{|c|c|}
\hline & Pair of phase masks \\
\hline $\begin{array}{l}\text { Cartesian coordinates. } \\
\text { Lateral displacements. }\end{array}$ & Polynomials [10] \\
\hline $\begin{array}{l}\text { Polar coordinates. } \\
\text { In plane-rotations. }\end{array}$ & $\begin{array}{c}\text { Trigonometric functions } \\
\text { of the polar angle } \\
{[11,12]}\end{array}$ \\
\hline $\begin{array}{l}\text { Polar coordinates. } \\
\text { In plane-rotations. }\end{array}$ & $\begin{array}{c}\text { Linear variations } \\
\text { of the polar angle } \\
\qquad 13,14]\end{array}$ \\
\hline
\end{tabular}

Here our aim to explore the use of two pairs of phase conjugated masks, working in tandem, for generating linear combinations of wave aberrations.

In the next section, we discuss a method for generating linear combinations of symmetric wave aberrations. Then,

*E-mail: jojedacas@ugto.mx we explore a method for incorporating asymmetric wave aberrations, into the linear combination.

For our present discussion we employ the following notation. As depicted in Fig. 1, we consider that we have two pairs of free form, refractive elements. The first pair is imaged, with magnification $\mathrm{M}=-1$, on the second pair. The Greek letters $\rho$ and $\varphi$ represent, respectively, the radial spatial frequency and the polar angle, at any of two conjugate planes. The cut-off spatial frequency is $\rho=\Omega$.

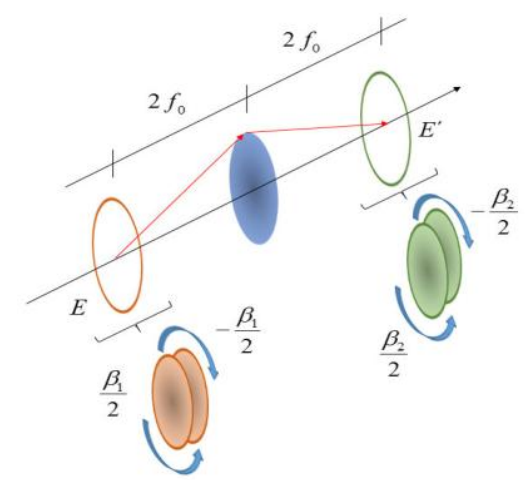

Fig. 1. Two vortex pairs in tandem.

Therefore, in the following equations, we assume that any complex amplitude transmittance is located inside a circular aperture, which is represented by the binary function $\operatorname{circ}(\rho / \Omega)$. This function is equal to unity inside a circle of radius $\rho=\Omega$. Otherwise, the $\operatorname{circ}(\rho / \Omega)$ is equal to zero. At the position of the first pair, the first element of this pair has the following complex amplitude transmittance

$$
P_{1}(\rho, \varphi)=\exp \left[i 2 \pi\left(\frac{\varphi}{2 \pi}\right) \sum_{m=0}^{M}\left(\frac{U_{m, 0}}{\lambda}\right)\left(\frac{\rho}{\Omega}\right)^{m}\right]
$$

In Eq. (1), the Greek letter $\lambda$ denotes the wavelength of the optical radiation. We have an aberration polynomial in the radial coordinate, aberration coefficients $U_{m, o}$, which reach their maximum value at $\rho=\Omega$, and $\varphi=2 \pi$. The complex amplitude transmittance of the second free form element is 


$$
P_{2}(\rho, \varphi)=P_{1}^{*}(\rho, \varphi)
$$

The complex amplitude transmittance of the second element is the complex conjugate of the first element. Next, we place together the two elements for setting the first pair. Then, we introduce an in-plane rotation (say by an angle $\alpha$ ) between the elements of the pair. Hence, for the first pair, the complex amplitude transmittance is

$$
\begin{aligned}
\mathrm{P}(\rho ; \alpha) & =P_{1}\left(\rho, \varphi+\frac{\alpha}{2}\right) P_{2}\left(\rho, \varphi-\frac{\alpha}{2}\right) \\
& =\exp \left[i 2 \pi\left(\frac{\alpha}{2 \pi}\right) \sum_{m=0}^{M}\left(\frac{U_{m, 0}}{\lambda}\right)\left(\frac{\rho}{\Omega}\right)^{m}\right] .
\end{aligned}
$$

It is apparent from Eq. (3) that the first pair generates a rotationally symmetric wavefront, with continuously variable optical path difference $\alpha$. Now, we consider that the same above analysis applies for the second pair; but now the wavefront aberration has a different polynomial expansion, and the value of the in-plane rotation angle is $\beta$. That is

$$
\begin{aligned}
\mathrm{Q}(\rho ; \beta) & =Q_{1}\left(\rho, \varphi+\frac{\beta}{2}\right) Q_{2}\left(\rho, \varphi-\frac{\beta}{2}\right) \\
& =\exp \left[i 2 \pi\left(\frac{\beta}{2 \pi}\right) \sum_{m=0}^{M}\left(\frac{V_{m, 0}}{\lambda}\right)\left(\frac{\rho}{\Omega}\right)^{m}\right] .
\end{aligned}
$$

Thus, the overall complex amplitude transmittance of both pairs, working in tandem, is

$$
\begin{aligned}
\mathrm{T}(\rho ; \alpha ; \beta) & =\mathrm{R}(\rho ; \alpha) \mathrm{Q}(\rho ; \beta) \\
= & \exp \left\{i \sum_{m=0}^{M}\left[\alpha\left(\frac{U_{m, 0}}{\lambda}\right)+\beta\left(\frac{V_{m, 0}}{\lambda}\right)\right]\left(\frac{\rho}{\Omega}\right)^{m}\right\}
\end{aligned}
$$

It is clear from Eq. (5) that we have obtained a linear combination of two wavefront aberrations, with weighting factors $\alpha$ and $\beta$. In Fig. 2 we illustrate our general result, by considering the generation of Zernike spherical aberration, say in the first pair. On the left-hand side of Fig. 2, we show the 3-D plots. On the right hand side, we display the contours of the phase delays. The images along Fig. 2 a) describe the first term of Eq. (3), for $\alpha=1$, while the images along Fig. 2 b) describe the second term of Eq. (3), for $\alpha=1$. Along Fig. 2 c), we display the generated wavefront.

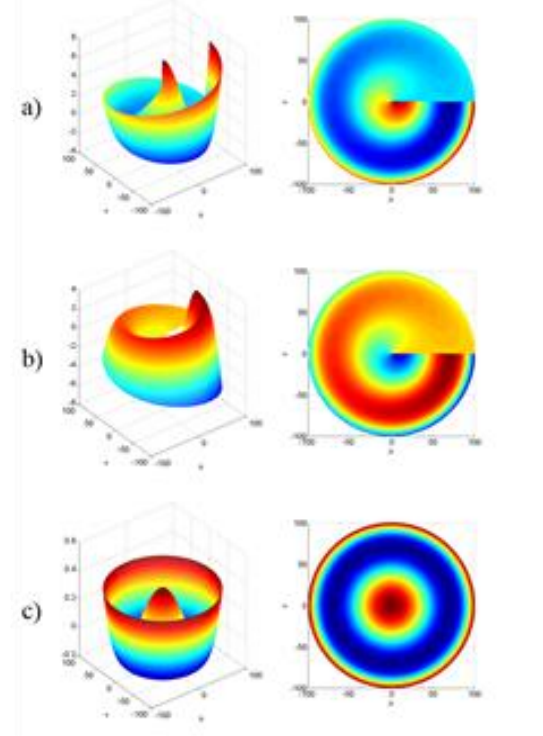

Fig. 2. The generation of Zernike's spherical aberration.

Other possibility is obtained if in Eqs. (3)-(4) we set

$$
\mathrm{U}(\rho)=\left(\frac{W_{6,0}}{\lambda}\right)\left(\frac{\rho}{\Omega}\right)^{6} ; \text { and } \quad V(\rho)=\left(\frac{W_{4,0}}{\lambda}\right)\left(\frac{\rho}{\Omega}\right)^{4} .
$$

Hence, the overall complex amplitude transmittance, in Eq. (5), becomes

$\mathrm{T}(\rho ; \alpha ; \beta)=\exp \left\{i\left[\alpha\left(\frac{\rho}{\Omega}\right)^{6}\left(\frac{W_{6,0}}{\lambda}\right)+\beta\left(\frac{W_{4,0}}{\lambda}\right)\left(\frac{\rho}{\Omega}\right)^{4}\right]\right\}$.

Of course, there is a myriad of possible combinations, which are beyond our present scope. In what follows we incorporate the presence of asymmetric wavefronts, in the proposed linear combination.

Now, for the sake of simplicity in our discussion, we consider that the complex amplitude transmittance of the first free form element, in the second pair, is

$$
Q_{1}(\rho, \varphi)=\exp \left[i 2 \pi\left(\frac{W_{m, n}}{\lambda}\right)\left(\frac{\rho}{\Omega}\right)^{2 m+1} \cos (n \varphi)\right] .
$$

As before, for the second element of the first pair, the complex amplitude transmittance is the complex conjugate of equation 8 . If we place together the two elements of the second pair, and we introduce an in-plane rotation between these elements, the complex amplitude transmittance is 
$\mathrm{Q}(\rho, \varphi ; \beta)=\exp \left[i 2 \pi\left(\frac{2 W_{m, n} \sin \left(\frac{n \beta}{2}\right)}{\lambda}\right)\left(\frac{\rho}{\Omega}\right)^{2 m+1} \sin (n \varphi)\right]$

It is apparent from Eq. (9) that by properly selecting the value of $\beta$, one is able to change continuously the aberration coefficient $\mathrm{W} m, \mathrm{n}$, by the factor $2 \sin (\mathrm{n} \beta / 2)$. In Fig. 3, we illustrate the generation of Seidel primary coma, $\mathrm{m}=1, \mathrm{n}=1$.

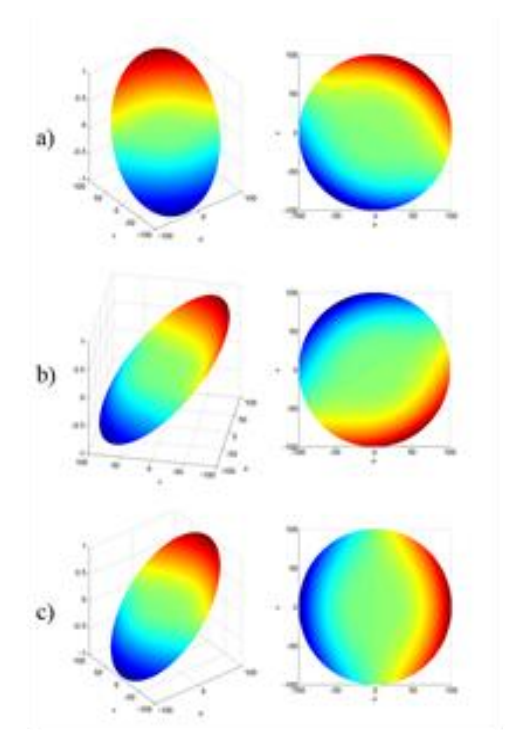

Fig. 3. Same as Fig. 2 but for Seidel primary coma.

Finally, by taking into account the results in Eqs. (3) and (9), one can obtain that for the two pairs working in tandem, the overall complex amplitude transmittance is a linear combination of symmetric aberrations with asymmetric aberrations. The weighting factors are $\alpha$ and twice the value of $\sin (n \beta / 2)$. That is,

$T(\rho, \varphi ; \alpha ; \beta)=\exp \left\{\begin{array}{l}i \alpha \sum_{m=0}^{M}\left(\frac{U_{m, 0}}{\lambda}\right)\left(\frac{\rho}{\Omega}\right)^{m} \\ +i 2 \pi\left(\frac{2 W_{m, n} \sin \left(\frac{n \beta}{2}\right)}{\lambda}\right)\left(\frac{\rho}{\Omega}\right)^{2 m+1} \sin (n \varphi)\end{array}\right\}$

We recognize that another possibility is to generate different asymmetric aberrations, in each pair, and the obtaining a linear combination of two different asymmetric aberrations.
In conclusion, we have discussed an optical method for (9) generating a linear combination of either symmetric or asymmetric wave aberrations. The proposed method uses two pairs of phase masks, which work in tandem. At each pair, the complex amplitude transmittance of the first element of the pair is the complex conjugate of the second element. After introducing an in-plane rotation between the elements of any pair, one can change continuously the weighting factor of a given wavefront. Since the two pairs work in tandem, then by superimposing the wavefronts of each pair, one can generate a linear combination of them. The weighting factors of the linear combination are related to the inplane rotation angles, which are represented by the parameters $\alpha$ and $\beta$. Hence, the weighting factors can be changed continuously. We have included some simple numerical simulations, for illustrating the proposed method.

We gratefully acknowledge the financial support of CoNaCyT, research project 157673, CA40120112. Ana Laura Barragan has a student research scholarship from CoNaCyT. We thank the reviewers for their useful comments.

\section{References}

[1] D.L. Fridge, J. Opt. Soc. Am. 50, 87 (1960).

[2] A. Buchroeder, R. Brian Hooker, Appl. Opt. 14, 2476 (1975).

[3] I. Kitajima, British Patent 250, 268 (July 29, 1926).

[4] A.W. Lohmann, British Patent 998, 191 (May 29, 1964).

[5] A.W. Lohmann, French Patent 1,398,351 (June 10, 1964).

[6] A.W. Lohmann, Italian Patent 727, 848 (June 19, 1964).

[7] A.W Lohmann, Appl. Opt. 9, 1669 (1970).

[8] L.W. Alvarez, U.S. Patent 3,305,294 (December 3, 1964).

[9] L.W. Alvarez, W.E. Humphrey, U.S. patent 3,507,565 (April 21,1970).

[10] I.A. Palusinski, J.M. Sasián, J.E. Greivenkamp, Appl. Opt. 38, 86 (1999).

[11] E. Acosta, Salvador Bará, J. Opt. Soc. Am. A 22, 1993 (2005).

[12] E. Acosta, J. Sasián, Opt. Express 19, 13171 (2011).

[13] J. Ojeda-Castaneda, Proc. SPIE 8833, 883306 (2013).

[14] J. Ojeda-Castaneda, S. Ledesma, C.M. Gómez-Sarabia, Phot. Lett. Poland 5, 20 (2013). 